\title{
ON EXTENSIONS OF SYMMETRIC OPERATORS
}

\author{
NAMig J. GULiYEV
}

Abstract. We give an explicit description of all minimal self-adjoint extensions of a densely defined, closed symmetric operator in a Hilbert space with deficiency indices $(1,1)$.

Mathematics subject classification (2010): 47A20, 47B25.

Keywords and phrases: Symmetric operator, self-adjoint extension, generalized resolvent.

\section{REFERENCES}

[1] C. Bartels, S. Currie, M. Nowaczy K, and B. A. Watson, Sturm-Liouville problems with transfer condition Herglotz dependent on the eigenparameter: Hilbert space formulation, Integral Equations Operator Theory 90 (2018), no. 3, Art. 34, 20 pp.

https://arxiv.org/abs/1804.07149.

[2] J. Behrndt ANd F. PhilipP, Finite rank perturbations in Pontryagin spaces and a Sturm-Liouville problem with $\lambda$-rational boundary conditions, Indefinite inner product spaces, Schur analysis, and differential equations, Birkhäuser/Springer, Cham, 2018, pp. 163-189.

[3] V. M. BRUK, A certain class of boundary value problems with a spectral parameter in the boundary condition (Russian), Mat. Sb. (N.S.) 100(142) (1976), no. 2, 210-216.

[4] V. DerKach, Boundary triplets, Weyl functions, and the Krein formula, Operator theory, Springer, Basel, 2015, pp. 183-218.

[5] B. Friedman, Principles and techniques of applied mathematics, John Wiley \& Sons, Inc., New York, 1956.

[6] C. T. Fulton, Two-point boundary value problems with eigenvalue parameter contained in the boundary conditions, Proc. Roy. Soc. Edinburgh Sect. A 77 (1977), no. 3-4, 293-308.

[7] F. Gesztesy and E. Tsekanovskit, On matrix-valued Herglotz functions, Math. Nachr. 218 (2000), 61-138, https://arxiv.org/abs/funct-an/9712004.

[8] N. J. GuliYev, Essentially isospectral transformations and their applications, Ann. Mat. Pura Appl. (4), to appear, https://arxiv .org/abs/1708.07497.

[9] M. G. KREIN, On Hermitian operators with deficiency indices one (Russian), Dokl. Akad. Nauk SSSR 43 (1944), 339-342.

[10] M. A. NAIMARK, Spectral functions of a symmetric operator (Russian), Izv. Akad. Nauk SSSR. Ser. Mat. 4 (1940), no. 3, 277-318.

[11] M. A. NAIMARK, On spectral functions of a symmetric operator (Russian), Izv. Akad. Nauk SSSR. Ser. Mat. 7 (1943), no. 6, 285-296.

[12] K. SCHMÜDGEN, Unbounded self-adjoint operators on Hilbert space, Springer, Dordrecht, 2012.

[13] A. V. Shtraus, On spectral functions of differential operators (Russian), Izv. Akad. Nauk SSSR. Ser. Mat. 19 (1955), no. 4, 201-220. 